ARTICLE

\title{
Effect of Air Condition on Alpha-radioactivity Measurement by Airflow Pulse Ionization Chamber
}

\author{
Naoki ZUSHI*, Yohei IHARA, Keisuke KABASHIMA, Fuminobu SATO, Yushi KATO \\ and Toshiyuki IIDA \\ Graduate School of Engineering, Osaka University, Yamada-oka, Suita, Osaka 565-0871, Japan
}

\begin{abstract}
A twin-type parallel-plate airflow pulse ionization chamber (APIC) was made for the examination of effects of air conditions on the measurement of alpha-radioactivity. Its symmetrical structure was effective for the reduction of common-mode noises in the alpha-ray measurement. The response of the APIC for ${ }^{241} \mathrm{Am}$ alpha-rays was measured for various air conditions. It was ascertained from pulse shape observation with a digital oscilloscope that lpha-ray-induced pulses had very slow risetime because of low mobility of negative ions in place of electrons. It was also confirmed from measurement of pulse height distributions that alpha-ray detection signals could be sufficiently discriminated from background noises. An experiment with a mantle containing thorium demonstrated that airborne alpha-radioactivity could be well measured with the APIC system.
\end{abstract}

\section{KEYWORDS: airflow pulse ionization chamber, alpha-radioactivity measurement, air condition}

\section{Introduction}

Radon and its progeny are known as the main radio nuclides distributed in nature. They easily enter into a human body and become the main cause of the internal exposure. Hence, in order to evaluate such internal exposure, various kinds of instruments and methods have been developed for the measurement of radon concentration in air.

The ionization chamber is a well-known tool for radiation detection and can be easily and inexpensively made because its structure is generally simple. Also, the pulse ionization chamber (PIC) detects each ionizing radiation as one pulse signal. A PIC has been used for the measurement of alpha-radioactivity of a sample set in the detector ${ }^{1-2)}$, and has also been improved for the measurement of radon concentration $^{3)}$.

For measurement of alpha-radioactivity in air, the ionization chamber system needs to be constructed in consideration of effects of impurities in ionization gas on charge collection process. For example, it is well known that airborne fine particles catch electrons generated by ionization and decrease charge mobility. This phenomenon has been applied to aerosol monitoring ${ }^{4}$. Similarly, several kinds of molecules decrease charge mobility because of electron attachment. Air includes detrimental impurities such as aqueous vapor and fine particles besides nitrogen and oxygen, so that the shape and amplitude of pulse signals from an airflow PIC (APIC)vary with air condition.

The purpose of this study is to demonstrate feasibility of alpha-radioactivity measurement by an APIC. It is essential for suitable signal processing to evaluate effects of air condition on charge collection process. In this study, a

*Corresponding Author, E-mail: zushi@nf.eie.eng.osaka-u.ac.jp (C) 2012 Atomic Energy Society of Japan, All Rights Reserved. twin-type parallel-plate APIC was made for the examination of air-condition-dependent effects on the alpha-radioactivity measurement, which were measured mainly by the use of a digital oscilloscope and a multi-channel pulse height analyzer (MCA).

\section{Materials and Methods \\ 1. Airflow Pulse Ionization Chamber}

A schematic representation of the whole APIC system is shown in Figure 1. The system was comprised of the APIC, modules for supplying different types of gas and detrimental impurities to the APIC and electronic circuits.

The APIC was made in an airtight plastic box covered with aluminum foil against electromagnetic noises. The box, of which size is $120 \mathrm{~mm} \times 160 \mathrm{~mm} \times 90 \mathrm{~mm}$, includes a twin-type parallel-plate APIC, i.e., four electrodes. The size of each electrode is $50 \mathrm{~mm} \times 100 \mathrm{~mm}$. The two central plates are high voltage electrodes, and the other two are signal electrodes. A small hole was bored in one of the high voltage electrodes and a standard ${ }^{241} \mathrm{Am}$ alpha-ray source was placed behind the electrode with the hole. The ${ }^{241} \mathrm{Am}$ source emitted 5.49 MeV alpha-rays at a rate of about $40 \mathrm{~Bq}$. The influence of low energy gamma-rays and $\mathrm{X}$-rays from the source was negligible in the measurement of alpha-rays, because the alpha-rays generated much larger amount of pulsed charge than the low energy gamma-rays and X-rays in the APIC.

External noises can deteriorate the signal-to-noise ratio and energy resolution in the alpha-ray measurement because of high sensitivity of charge sensitive amplifiers (CSAs). The twin-type APIC were almost equally affected by external noises induced by mechanical vibration, commercial electricity and others. Such common-mode noises were efficiently reduced by the use of a differential amplifier (DA). 


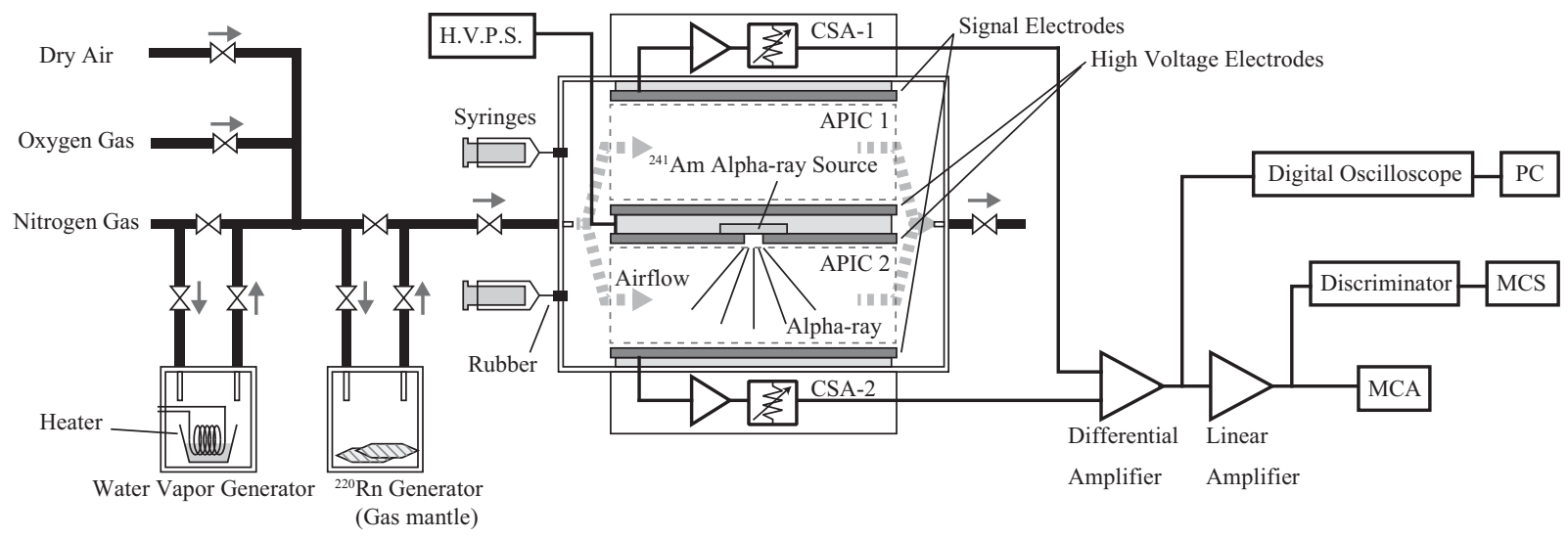

Fig. 1 Schematic drawing of airflow pulse ionization chamber together with gas supply system and electronic circuits.

A CSA was located directly above each signal electrode across an insulation plate for the reduction of the input capacitance, which degrades signal-to-noise ratio in the alpha-ray measurement. The CSAs were also covered with aluminum foil against electromagnetic noises.

Output signals from each CSA were fed to a DA for the reduction of common-mode noises. The gain of the CSA-2 (for detection of alpha-rays from the ${ }^{241} \mathrm{Am}$ source)and the other CSA-1 was adjusted for the precise cancellation of common-mode noises. Output signals from the DA were amplified by a linear amplifier (LA) with appropriate time constant for the pulse shaping. Output signals from the LA were thereafter analyzed with the MCA and/or were counted with a multi-channel scaler (MCS) through a pulse height discriminator. Pulse shapes of output signals from the DA were also observed with a digital oscilloscope (DO, LT584, Lecroy) followed by a personal computer (PC). We made all the electronic circuits except the DO and PC for the present experiments.

The risetime of output pulses from the CSA was, on the whole, the order of millisecond for the APIC. Thus, the shaping time of the LA was optimally adjusted for such slow pulses.

\section{Gas Supply System}

Nitrogen and oxygen gas, dry air and several detrimental components were prepared for the examination of the characteristics of the APIC. The small amount of the detrimental component was injected into the APIC with a set of syringes. Water vapor was also produced by the use of a heater in a nitrogen gas container. In addition, silica gel was set in an air supply module for the investigation of the humidity effect.

As a source of airborne alpha-radioactivity, a gas mantle (STG-281, SOTO)for a camping lantern was put into a container with valves as shown in Figure 1. Some kinds of gas mantle contain ${ }^{232} \mathrm{Th}$ and generate ${ }^{220} \mathrm{Rn}$ and its daughter nuclides, and the gas mantle can be well used as ${ }^{220} \mathrm{Rn}$ (thoron) sources. An experiment with such ${ }^{220} \mathrm{Rn}$ gas was carried out for the confirmation whether airborne alpha-radioactivity could be measured with the APIC system or not.

\section{Experimental}

First of all, pulse shapes and pulse height distributions of the APIC filled with pure nitrogen gas were measured for alpha-rays from the ${ }^{241} \mathrm{Am}$ source placed behind the high voltage electrode, to which a high voltage of $-2.5 \mathrm{kV}$ was applied. The gap between the signal and high voltage electrodes was set to be $4 \mathrm{~cm}$. Nitrogen has low electron attachment coefficient and is considered to be one of the most suitable gas for APIC. Then, in this study, a decline in the fundamental characteristics of the nitrogen PIC was examined through the injection of the detrimental component into the nitrogen gas. Pulse shapes and pulse height distributions of the APIC including the detrimental component for alpha-rays were compared to those of the pure nitrogen PIC.

The humidity effects on the APIC characteristics were examined through the injection of water vapor into the nitrogen APIC or the injection of the wet air into the dry air APIC. Furthermore, the effects of structural (electrode gap)and electrical (applied voltage) conditions of the APIC were examined in consideration of the breakdown caused due to a too high electric field. And $\mathrm{a}^{220} \mathrm{Rn}$ alpha-radioactivity measurement was preliminarily performed with the APIC of which structural and electrical conditions were optimally adjusted.

\section{Results and Discussion}

\section{Response of Pulse Ionization Chamber to ${ }^{241} \mathrm{Am}$ Alpha-rays}

Figure 2 shows examples of typical pulse shapes from the PIC filled with different types of gas for ${ }^{241} \mathrm{Am}$ alpha-rays, which wereoutput pulse shapes from the CSA and were observed in the averaging mode of the digital oscilloscope. The integration time constant of the CSA was $10 \mathrm{~ms}$. In the case of the pure nitrogen gas, the pulse immidiately rose and decayed in accordance with the time constant of $10 \mathrm{~ms}$. On the other hand, the pulse shapes measured foroxygen gas and air had slow risetime of about 5 ms. Furthermore, their pulse amplitude was about $15 \%$ smaller than that measured for thepure nitrogen gas. The difference in the risetime of the pulses between the nitrogen and oxygen gas or air is related to the types of negative 


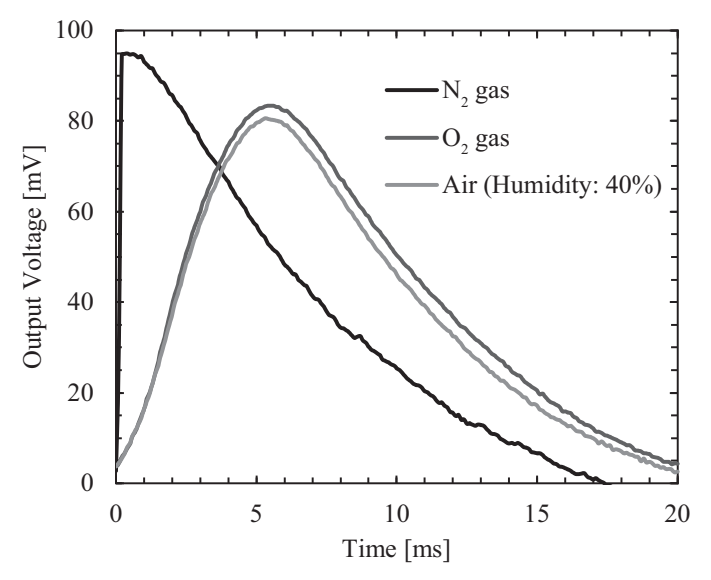

Fig. 2 Typical pulse shapes from APIC filled with different types of gas for ${ }^{241} \mathrm{Am}$ alpha-rays.

charge carriers, which approach the signal electrode and play a large role in the pulse formation. The negative high voltage was applied to the high voltage electrode. The fast risetime observed for thepure nitrogen gas means that the negative charge was carried by electrons which could move quickly. In the case of the oxygen gas and air, oxygen molecules easily catch electrons and are apt to become negative ions, so that the slow risetime of the pulses was caused due to the low mobility of such negative charge carriers (not electrons but ions).

Water was evaporated with an electric heater to make water vaporin nitrogen gas. It was confirmed from an experiment on the water vapor effect that the risetime of alpha-ray detection pulses was sensitively slowed down by the injection of water vapor into the pure nitrogen gas. This result is also considered to be due to the formation of negative ions. As for air, on the other hand, no large humidity effect was observed, for example, there was no large change in the pulse shape for the ${ }^{241} \mathrm{Am}$ alpha-ray detection in the humidity range below $62 \%$. This is probably because the humidity effect was under the large degradation effect due to the large amount of oxygen molecules in air.

Figure 3 shows examples of typical pulse height distributionsof the APIC filled with different types of gas for ${ }^{241} \mathrm{Am}$ and ${ }^{220} \mathrm{Rn}$ alpha-rays. It is clear from the figure that the alpha-ray detection signals can be successfully discriminated from noises and that the alpha-radioactivity in air can be satisfactorily measuired with the APIC filled with normal air, although its energy resolution is inferior to the case of the pure nitrogen gas. The pulse height distribution for ${ }^{220} \mathrm{Rn}$ related alpha-rays (dotted line) ranged broadly because the alpha-ray emission spots were not fixed. Different energy alpha-rays from ${ }^{220} \mathrm{Rn}$ daughter nuclides may broaden the energy spectrum. Even in such a case, a appropriate pulse height discriminaton level can be easily set for the measurement of the alpha-radioactivity.

\section{Effect of Electrode-gap}

The performance of the APIC is not only affected by air conditions but basically depends on its structure and the

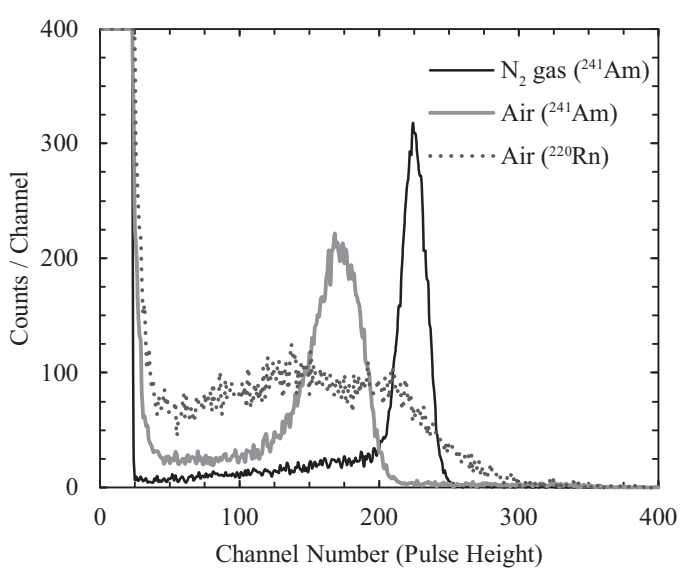

Fig. 3 Typical pulse height distributions of APIC filled with different types of gas for alpha-rays.

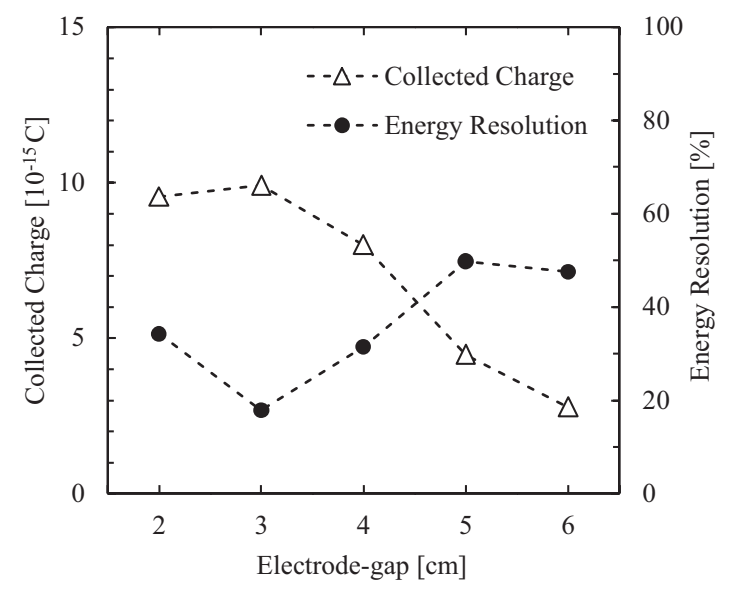

Fig. 4 Relation between electrode-gap and response of APIC for ${ }^{241}$ Am alpha-rays.

electrical bias condition.It is desirable for pulse signals from the APICto properly have fast components, which leads to better signal-to-noise ratio in the pulse measurement. Therefore, a higher bias voltage can collect electric charge quickly and produces fast pulse signals, in other words, it is desirable to keep the bias voltage as high as possible.

However, a too high bias voltage easily causes electrical breakdown, which is also affected by humidity and other ambient conditions.

The gap between the signal and high voltage electrodes is also one of the essential factors to characterize the APIC.In the case of a too narrow electrode-gap, though a strong electric field can be formed by a low bias voltage, a sensitive volumebecomes small andtherfore it becomes difficult for gas in the APIC to absorb the whole energy of an alpha-ray, which results in the degradation of the energy resolution. The APIC with a too wide electrode-gap has difficulty in producing alpha-ray detection signals with good pulse shapes.

Figure 4 shows relation between the electrode-gap and response characteristics of the APIC for ${ }^{241} \mathrm{Am}$ alpha-rays. The best charge collection efficiency and the best 


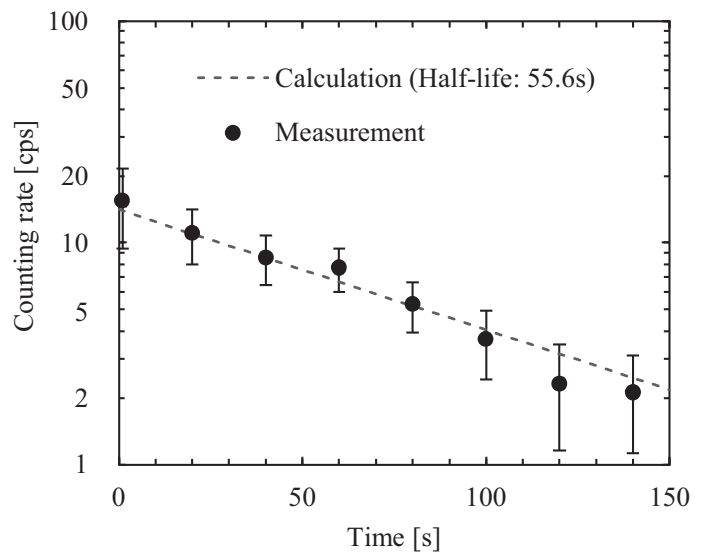

Fig. 5 Change in counting rate of ${ }^{220} \mathrm{Rn}$-related alpha-rays measured with APIC system.

energy resolution were found for the electrode gap of $3 \mathrm{~cm}$. However, we set the electrode-gap to $4 \mathrm{~cm}$ in present APIC systemin consideration of a largersensitive volume for the absorption of the whole energy of alpha-rays from airborne radionuclides.

\section{Measurement of ${ }^{220}$ Rn-related Alpha-rays}

A preliminary airborne alpha-radioactivity measurement was carried out by the present APIC system. ${ }^{220} \mathrm{Rn}$ contained air was injected into the APIC. The result of pulse height distribution of the APIC for ${ }^{220} \mathrm{Rn}$-related alpha-rays was shown in Figure 3.

In this ${ }^{220} \mathrm{Rn}$-related alpha-ray measurement, the change in the alpha-ray counting rate was measured with the MCS through the pulse height discriminator. All valves of the APIC were closed after the injection of the ${ }^{220} \mathrm{Rn}$-contained air.

Figure 5 represents the decay in the alpha-ray counting rate measured with the APIC system. The measured result on the decay curve agreed with the well-known half-life of ${ }^{220} \mathrm{Rn}(55.6 \mathrm{sec})$, which indicates that the airborne alpha-radioactivity in air can be continuously measured with the present APIC system.

\section{Conclusion}

The airflow pulse ionization chamber (APIC) was made for the examination of effects of air conditions on the measurement of alpha-radioactivity such as radon. The symmetrical structure of the twin-type parallel-plate APIC effectively eliminated external common-mode noises. The shapes of response pulses and the pulse height distributions of the APIC for ${ }^{241} \mathrm{Am}$ alpha-rays were measured for various air conditions. It was ascertained from the pulse shape observation for the APIC that pulses induced by alpha-rays in air had very slow rise time because of low mobility of negative ions in place of electrons. It was also confirmed from the measurement of pulse height distributions that alpha-ray detection signals could be satisfactorily discriminated from background noises. The experiment with a mantle containing thorium demonstrated that airborne alpha-radioactivity could be well measured with the present APIC system.

\section{References}

1) L. Johansson, B. Roos, C. Samuelsson, "Alpha-particle spectrometry of large-area samples using an open-flow pulse ionisation chamber" Appl. Radiat. Isot., 43(1-2), 119-125 (1992)

2) Y.M. Gavrilyuk, A.M. Gangapshev, V.V. Kazalov, V.V. Kuz'minov, S.I. Panasenko, S.S. Ratkevich, "An ion pulse ionization chamber for spectrometric measurements of low surface $\alpha$ activities" Instrum. Exp. Tech., 52(2), 173-182 (2009)

3) A. Froňka, L. Moučka, M. Jeřábek, "Detection properties of a measuring system for a continuous soil radon concentrations monitoring" Radiat. Prot. Dosim., 130(1), 56-59(2008)

4) N.G. Bernigau, H.O. Luck, "The principle of the ionization chamber in aerosol measurement techniques-a review" $J$. Aerosol Sci., 17(3), 511-515(1986) 